\title{
Kidney-specific cadherin, a specific marker for the distal portion of the nephron and related renal neoplasms
}

\author{
Steven S Shen ${ }^{1}$, Bhuvaneswari Krishna ${ }^{2}$, Rukmini Chirala ${ }^{1}$, Robert J Amato ${ }^{3}$ and \\ Luan D Truong ${ }^{1,4}$ \\ ${ }^{1}$ Department of Pathology, The Methodist Hospital, Baylor College of Medicine, Houston, TX, USA; ${ }^{2}$ Veterans \\ Affairs Medical Center, The Methodist Hospital, Baylor College of Medicine, Houston, TX, USA; ${ }^{3}$ Scott \\ Department of Urology, The Methodist Hospital, Baylor College of Medicine, Houston, TX, USA and \\ ${ }^{4}$ Department of Medicine, The Methodist Hospital, Baylor College of Medicine, Houston, TX, USA
}

\begin{abstract}
Renal cell neoplasms are presumably derived from different cell types of the nephron. Clear cell and papillary renal cell carcinoma (RCC) are thought to be of proximal tubular origin, whereas oncocytoma and chromophobe RCC are derived from intercalated cells of distal nephron. A few molecules, such as RCC marker and CD10, have been shown to be markers for clear cell RCC and papillary RCC. Such markers are not yet available for renal tumors presumably of the distal nephron. The expression of kidney-specific (Ksp) cadherin, a recently cloned gene thought to be transcribed exclusively in the kidney, was studied in normal human kidney, as well as in 105 primary renal neoplasms, including 42 clear cell RCC, 30 papillary RCC, 13 chromophobe RCC, and 20 oncocytomas. The expression patterns were compared with those of RCC marker. The Ksp-cadherin expression was noted preferentially in distal convoluted tubules with a basolateral membrane stain in normal kidney. All 13 chromophobe RCC and 19 of 20 oncocytomas showed diffuse and strong immunoreactivity for Ksp-cadherin, while only $14 \%$ clear cell RCC and $13 \%$ papillary RCC showed focal positivity. The RCC marker expression was detected in $85 \%, 98 \%, 15 \%$ and $0 \%$ of clear cell RCC, papillary RCC, chromophobe RCC, and oncocytoma, respectively. A few clear cell RCC and papillary RCC showed dual expression of both RCC marker and Kspcadherin, which appear to have distinct histologic features. These results demonstrated high sensitivity and specificity of Ksp-cadherin for distal convoluted tubules, which can be used as adjunct for diagnosis of chromophobe RCC.

Modern Pathology (2005) 18, 933-940. doi:10.1038/modpathol.3800373; Published online 21 January 2005
\end{abstract}

Keywords: kidney-specific cadherin; renal neoplasms; immunostain; chromophobe; oncocytoma

Renal neoplasms consist of heterogeneous groups of benign and malignant tumors. The four major subtypes are clear cell (conventional) renal cell carcinoma (RCC), papillary RCC, chromophobe RCC, and oncocytoma, which together account for approximately $90 \%$ of all renal neoplasms. Accurate diagnosis of these tumor types is important since their behavior, prognosis, and management are different. Although the majority of renal tumors do display characteristic light microscopic features that lead to a correct diagnosis, the morphologic overlap between tumors and the histologic heterogeneity

Correspondence: Dr SS Shen, MD, PhD, Department of Pathology, MS 205 The Methodist Hospital, 6565 Fannin Street, Houston, TX 77030, USA.

E-mail: stevenshen@tmh.tmc.edu

Received 4 October 2004; revised and accepted 3 December 2004; published online 21 January 2005 within a single tumor continue to create problems in the diagnosis and, particularly, the classification of renal neoplasms. Some histochemical stains and immunohistochemical markers are shown to be helpful in the differential diagnosis of these tumors. Hale's colloidal iron stain can support the diagnosis for chromophobe RCC, ${ }^{1,2}$ and immune markers such as vimentin, ${ }^{3,4} \mathrm{CD} 10,{ }^{5}$ parvalbumin ${ }^{6,7}$ E-cadherin, ${ }^{8}$ or RCC marker ${ }^{5,9}$ have been used to aid in the diagnosis and classification of renal neoplasms. These special studies are, however, quite limited. For example, although E-cadherin and parvalbumin are expressed by chomophobe RCC and oncocytoma, they also have wide tissue distribution and are positive in a wide range of neoplasms, as are vimentin and CD10. In contrast, although the RCC marker is quite kidney-specific, it is limited to proximal tubules and thus is often expressed by RCC thereof derived such as clear cell or papillary RCC, 
but usually negative in other types of renal neoplasms. ${ }^{5,9}$ Additional immunomarkers for renal neoplasms, especially for those of distal nephron origin, are desirable.

Kidney-specific (Ksp)-cadherin, a member of the cadherin family of calcium-dependent cell adhesion molecules, was recently identified as a specific marker for terminal differentiation of the basolateral membrane of renal tubular epithelial cells. ${ }^{10,11} \mathrm{Un}$ like most of the cadherins, which often have a widespread tissue distribution, Ksp-cadherin appears to have unique kidney-specific distribution, and within the kidney, it is observed predominantly in the distal portion of the nephron. ${ }^{11,12}$ The expression of Ksp-cadherin in human kidney tissue and renal cell neoplasm has not been evaluated.

In this study, we have evaluated and characterized the expression of Ksp-cadherin in normal human kidney tissue, as well as in the more common types of renal neoplasms using a recently developed, commercially available mouse monoclonal antibody and to compare its utility in the diagnosis and classification of these tumors with those of RCC marker.

\section{Materials and methods}

Cases originally diagnosed as clear cell RCC $(n=42)$, papillary RCC $(n=30)$, chromophobe RCC $(n=13)$, and renal oncocytoma $(n=20)$ were selected from the files of the Departments of Pathology, the Methodist Hospital and VA Medical Center, Houston, Texas (Table 1). All original slides from each case were reviewed by two of the authors (SS and LT) and tumors were reclassified according to the criteria developed in the 1997 consensus conference on renal cell neoplasm. ${ }^{13,14}$ Special attention was paid to the cases in which the original histologic classification was revised, those with features of more than one tumor type, and those that did not fit in any well-defined category. We also assessed the diagnostic value of combination of Ksp-cadherin and RCC marker in some cases.

Representative and consecutive sections from each tumor, most of which contained adjacent non-neoplastic tissue, were submitted to immunostain with the RCC marker (Vector Laboratories, Burlingame, CA, USA; dilution 1:10) and an anti-

Table 1 Histologic features of renal cell neoplasms

\footnotetext{
Clear cell RCC (42 cases)

Grade: 1 (3 cases), 2 (24 cases), 3 (16 cases), 4 (3 cases)

Papillary RCC (30 cases)

Grade: 1 (5 cases), 2 (17 cases), 3 (7 cases), 4 (1 cases)

Type 1 (26 cases), type 2 (4 cases)

Chromophobe RCC (13 cases)

Grade: 1 (1 case), 2 (5 cases), 3 (6 cases), 4 (1 case)

Typical type (11 cases), eosinophilic type (2 cases) Oncocytoma (19 cases)
}

body against Ksp-cadherin (Zymed Laboratories, San Francisco, CA, USA; dilution 1:75). Formalinfixed, paraffin-embedded tissue sections were used with a standard avidin-biotin-peroxidase complex technique after steamed heat-mediated antigen retrieval. The positive controls included normal kidney tissue and/or the non-neoplastic kidney tissue adjacent to the tumors. The negative controls included replacement of the primary antibody with nonimmune mouse serum. For each case, the visually estimated percentage of stained tumor cells and the staining intensity were independently evaluated and recorded by two of the authors (SS and LT).

\section{Results}

Formalin-fixed, paraffin-embedded tissue provided excellent staining with both the RCC marker and the Ksp-cadherin, with appropriate results for the positive and negative controls.

\section{Non-Neoplastic Kidney Tissue}

In normal kidney tissue, Ksp-cadherin expression was seen predominantly in medulla (Figure 1a, e) in contrast with cortical accentuation of RCC marker staining (Figure 1b, d). Strong expression of Kspcadherin was detected in distal convoluted tubules and thick Henle loops throughout their length in a unique basolateral pattern. There was focal very weak staining for proximal tubules and collecting ducts also in a basolateral pattern (Figure 1c, e). The glomeruli, stromal cells, vessels, and inflammatory cells were negative. The RCC marker was strongly expressed throughout the length of the proximal tubules in a 'brush-border' pattern, but was not seen in other cell types in the kidneys (Figure 1d and f).

In non-neoplastic but atrophic kidney tissue adjacent to the tumor, where light microscopic differentiation among different types of renal tubules became difficult, the atrophic tubules, however, can still be recognized and identified by their specific positive staining for RCC marker and Kspcadherin, although less intense.

\section{Renal Cell Neoplasms}

The overall results and those related to individual tumor types were summarized in Table 2. Kspcadherin was positive in $100 \%$ of chromophobe RCC and $95 \%$ of oncocytoma. The stain was distinctively membranous with accentuation at the basolateral aspect (Figure 2e and g). All except $14 \%$ of clear cell RCC and $13 \%$ of papillary RCC were negative for Ksp-cadherin (Figure 2a and c). The sensitivity for chromophobe RCC/oncocytoma was $97 \%$ and specificity was $86 \%$. The positive and negative predictive values were $76 \%$ and $98 \%$, respectively. RCC 



Figure 1 Staining of Ksp-cadherin (a, c, and e) and RCC marker (b, d, and f) in normal kidney (consecutive sections). (a) Diffuse Kspcadherin tubular staining in medulla contrasting with focal tubular staining in cortex and corticomedullary junction. (c) Only some cross-sections of distal convoluted tubules in the cortex show positive Ksp-cadherin staining. (e) Strong basolateral Ksp-cadherin staining is noted for the thick portion of Henle loops in medulla. (b) Diffuse tubular staining for the RCC marker in the cortex without staining in the medulla. (d) Diffuse RCC marker staining of the brush border of proximal tubules in the cortex. (f) Lack of RCC marker staining in the medulla.

marker was positive in $86 \%$ of clear cell RCC and $97 \%$ of papillary RCC with a predominantly apical distribution (Figure $2 \mathrm{~b}$ and $\mathrm{d}$ ). This marker was negative in $85 \%$ of chromophobe RCC and in all oncocytomas (Figure $2 \mathrm{f}$ and $\mathrm{h}$ ). Positive stain for either Ksp-cadherin or the RCC marker, however, 
Table 2 Immunostain of renal neoplasms for Ksp-cadherin (KSC) and RCC marker (RCC Ma)

\begin{tabular}{lccc}
\hline Histologic type & KSC+ & $\begin{array}{c}\text { RCC } \\
M a+\end{array}$ & $\begin{array}{c}\text { KSC+ or RCC } \\
M a+\end{array}$ \\
\hline $\begin{array}{l}\text { Clear cell RCC }(n=42) \\
\text { Papillary RCC }(n=30)\end{array}$ & $6(14 \%)$ & $36(86 \%)$ & $38(91 \%)$ \\
$\begin{array}{l}\text { Chromophobe RCC } \\
(n=13)\end{array}$ & $13(100 \%)$ & $29(97 \%)$ & $30(100 \%)$ \\
Oncocytoma $(n=20)$ & $19(95 \%)$ & $0(0 \%)$ & $13(100 \%)$ \\
\hline
\end{tabular}

was noted in $91-100 \%$ of tumors, depending on the histologic subtype.

Of $42(86 \%)$ clear cell renal cell carcinoma (Table 3), 36 were positive for RCC marker, among which, 31 were negative for Ksp-caherin. The RCC marker positive/Kap-cadherin negative tumors had 'classic' features of clear cell RCC with solid or acinar growth pattern, delicate branching vascular network, and abundant clear cytoplasm. Five RCC marker-positive tumors were also Ksp-cadherin positive in $20-70 \%$ of tumor cells, and each of them, although still best classified as clear cell RCC on review, displayed some distinctive histologic features characterized by a mixed tubulocystic or tubulopapillary architecture and tumor cells with voluminous clear and granular cytoplasm and prominent nucleoli (Figure 3a). The tumor showed typical basolateral staining for Kspcadherin and apical staining for RCC marker, and the staining for Ksp-cadherin and RCC marker appear to be in the same tumor cells (Figure $3 \mathrm{~b}$ and c). All five except one clear cell carcinoma with dual staining of Ksp-cadherin and RCC marker were Furhman's nuclear grade 3 or 4 and grossly extended to renal vein. Five clear cell carcinomas showed neither RCC marker nor Ksp-cadherin stains, among them three of them showed significant cystic component and the other two exhibited typical clear cell morphology. One tumor showed negative RCC marker stain, but positive for Ksp-cadherin. This tumor showed mixture of eosinophilic and clear cells.

Of $30(97 \%)$ papillary renal cell carcinomas, 29 were positive for RCC marker (Table 4). Of them, 26 were negative for Ksp-cadherin, and three were positive with $30-60 \%$ of cells staining. All three RCC marker-positive/Ksp-cadherin-positive tumors were type 2 papillary RCC, characterized by broad papillae and tumor cells with abundant eosinophilic cytoplasm and nuclear grade 3 or 4 (Figure 4a). These tumors also showed typical basolateral staining for Ksp-cadherin and apical staining for RCC marker in same tumor cells (Figure $4 \mathrm{~b}$ and c). One
RCC marker-negative/Ksp-cadherin-positive papillary RCC also showed type 2 morphology.

All 13 chromophobe RCC were positive for Kspcadherin in a mean of $70 \%$ tumor cells (Table 5). Two of them were also positive for RCC marker in $70 \%$ and $100 \%$ of tumor cells with a typical apical pattern. Both of them represented the eosinophilic variant of chromophobe renal cell carcinoma.

Of 20 oncocytomas, 19 were positive for Kspcadherin in a mean of $40 \%$ tumor cells. However, none of these tumors were positive for RCC marker (Table 6). A single oncocytoma was negative for both Ksp-cadherin and RCC marker. The tumor appears to have the typical oncocytoma morphology with predominantly solid and alveolar patterns.

\section{Discussion}

Cadherins are members of a superfamily of adhesion molecules, which are ubiquitously expressed and play a crucial role in cell-cell interaction and tissue homeostasis. Ksp-cadherin was initially identified and cloned from rabbit by Thompson et $a l^{10}$ as a novel cadherin that is expressed only in the kidney. The human and mouse isoforms of Ksp-cadherin were subsequently cloned and were also kidneyspecific. ${ }^{12} \mathrm{~A}$ monoclonal antibody against Kspcadherin is now commercially available. To our knowledge, this is the first report that systematically evaluates the expression of Ksp-cadherin in human kidney tissue and its role in the diagnosis and classification of renal neoplasms.

We have shown that: (1) The monoclonal antibody against Ksp-cadherin tested in this study provides excellent staining on routine formalin-fixed paraffin-embedded tissue and therefore may have practical application in diagnostic pathology. (2) In normal human kidney, Ksp-cadherin is localized to the basolateral portion of the renal tubular cells with a distinctive distribution along the nephron that is characterized by strong and diffuse staining for distal tubules and thick Henle loop, with less expression in collecting ducts. (3) Ksp-cadherin appears to be highly sensitive and specific markers for renal neoplasms of distal nephron derivation/ differentiation, for example, chromophobe RCC and oncocytoma.

In this study, we have investigated and compared the Ksp-cadherin expression in RCC with that of RCC marker, a specific marker for proximal tubules, to assess the histogenesis of RCC and to test the diagnostic utility of these markers separately or in combination. We found that although all major types of renal tumors variably express Ksp-cadherin, it is a highly sensitive and specific marker for chromo-

Figure 2 The immunohistochemical staining of Ksp-cadherin (a, $\mathbf{c}, \mathbf{e}, \mathbf{g})$ and RCC marker $(\mathbf{b}, \mathbf{d}, \mathbf{f}, \mathbf{h})$ in clear cell RCC (a, b), papillary RCC $(\mathbf{c}, \mathbf{d})$, chromophobe RCC $(\mathbf{e}, \mathbf{f})$, and oncocytoma $(\mathbf{g}, \mathbf{h})$. An apical pattern is noted for the RCC marker, whereas a cell membrane pattern is evident for Ksp-cadherin. 


Table 3 Immunostain of 42 clear cell RCC

\begin{tabular}{llr}
\hline & KSC+ & \multicolumn{1}{c}{ KSC- } \\
\hline RCC+ & $5(12 \%)$ & $31(74 \%)$ \\
RCC- & $1(2 \%)$ & $5(12 \%)$ \\
\hline
\end{tabular}

RCC $=$ RCC marker; KSC $=$ Ksp-cadherin
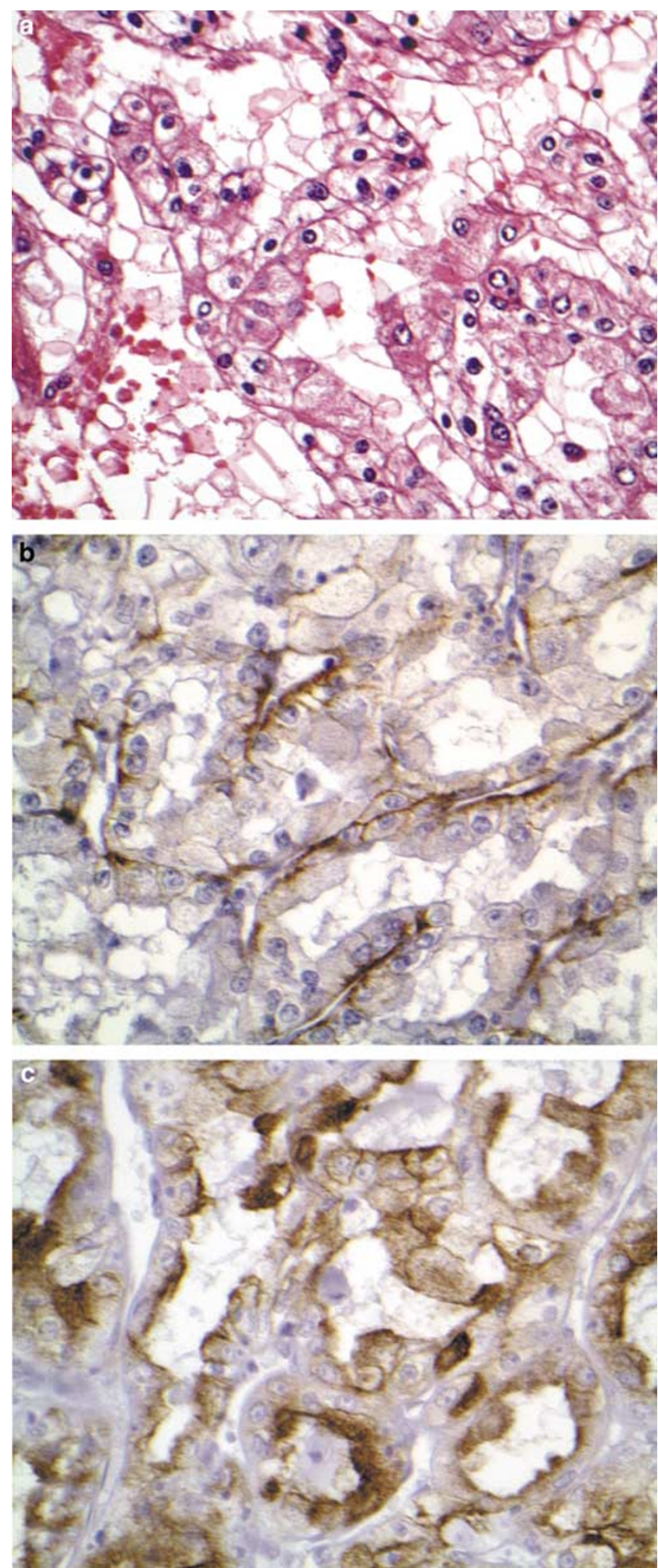

Table 4 Immunostain of 30 papillary RCC

\begin{tabular}{llc}
\hline & KSC+ & KSC- \\
\hline RCC+ & $3(10 \%)$ & $26(87 \%)$ \\
RCC- & $1(3 \%)$ & $0(0 \%)$ \\
\hline
\end{tabular}

See footnote in Table 3.

phobe RCC and oncocytoma. Most chromophobe RCC can be readily diagnosed by light microscopic features, but this diagnosis may sometimes require ancillary studies. Although E-cadherin and parvalbumin have been shown to be markers for chromophobe RCC and oncocytoma, there are limitations. E-cadherin, which has been detected in vertebrate kidney and appears to involve tubular cell genesis differentiation, has a widespread extrarenal distribution and has been shown in almost all solid tumors, including that of prostate, lung, breast, and urinary bladder. ${ }^{15,16}$ Similarly, parvalbumin, a calcium-binding protein with a major role in calcium homeostasis, is detected in many metabolically active cell types such as muscle, neuron, and endocrine glands as well as their related tumors. ${ }^{17,18}$ In contrast, Ksp-cadherin, is limited to the kidney. Previous immunohistochemical and Northern hybridization studies have failed to show its expression in other organs including lung, prostate, pancreas, skin, muscle, liver, stomach and intestine. ${ }^{12}$ Our findings that all chromophobe RCC and almost all oncocytomas express Ksp-cadherin not only support the theory of distal nephron origin of these renal tumors but also introduce an accurate marker for their diagnosis.

The current study confirms RCC marker as a sensitive and specific marker for papillary and clear cell carcinoma and further suggests enhancement of it use in combination with Ksp-cadherin. Although 80 and $26 \%$ of RCC, regardless of histologic types, are positive for RCC marker and Ksp-cadherin, respectively, $95 \%$ of them express one of these two markers. Our observations suggest a combination of these two markers to cover both proximal and distal line of differentiation and thus improve the diagnosis of both primary and metastatic renal tumors.

This study provides some interesting insights into the histogenesis of RCC and its morphologic heterogeneity even within the same tumor. Although the majority of RCC in this study showed either RCC marker or Ksp-cadherin along the line of the proximal vs distal nephron differentiation, well

Figure 3 Clear cell RCC with dual expression of Ksp-cadherin and the RCC marker. (a) H\&E stain showing an area with tubulocystic or tubulopapillary architecture and cells with clear and eosinophilic cytoplasm. (b) Tumor cells in this area express Ksp-cadherin in a basolateral pattern. (c) Tumor cells from the same area express the RCC marker in a luminal/apical staining of tumor cells. 



Figure 4 Papillary RCC with dual expression of Ksp-cadherin and the RCC marker. (a) H\&E stain showing papillary architecture and lined by cells with abundant eosinophilic cytoplasm, moderately sized nuclei and inconspicuous nucleoli. (b) Ksp-cadherin stain showing basolateral membrane staining of tumor cells. (c) RCC marker stain showing the luminal/apical staining of tumor cells in the same area.

accepted in the known histogenetic scheme of renal tumors, dual staining, albeit with one marker still predominant, was noted in up to $12 \%$ of RCC. We also noted that the Ksp-cadherin positive/RCC
Table 5 Immunostain of 13 chromophobe RCC

\begin{tabular}{lrr}
\hline & \multicolumn{1}{c}{$K S C+$} & $K S C-$ \\
\hline RCC+ & $2(15 \%)$ & $0(0 \%)$ \\
RCC- & $11(85 \%)$ & $0(0 \%)$ \\
\hline
\end{tabular}

See footnote in Table 3.

Table 6 Immunostain of 20 oncocytomas

\begin{tabular}{lcc}
\hline & $K S C+$ & $K S C-$ \\
\hline RCC+ & $0(0 \%)$ & $0(0 \%)$ \\
RCC- & $19(95 \%)$ & $1(5 \%)$
\end{tabular}

See footnote in Table 3.

marker-positive clear cell RCC appears to have a distinct morphology characterized by tubulocystic or tubulopapillary architecture, higher nuclear grade, and eosinophilic cytoplasm. Whether these tumors represent a unique subset of neoplasm cytogenetically remains to be studied. Interestingly, we also found that four of five clear cell carcinoma with dual staining were of Furhman's nuclear 3-4 and grossly extend to the renal vein, raising the possibility of prognostic importance of these histologic and immunohistochemical features of these tumors. It will be important to verify these findings in larger number of such cases in the future. Similarly, we found that although almost all papillary RCC were, as expected, positive for RCC marker, those also with a positive Ksp-cadherin staining interestingly were of type 2 morphology. This observation is consistent with a recent study showing that most papillary RCC, which displayed Ecadherin, were of type $2 .^{8}$ These observations suggested that a significant portion of RCC display a dual differentiation, which may or may not be appreciated at light microscopic level. The explanation for dual differentiation within a single tumor is not clear. One possibility is that all renal neoplasms may derive from pluripotential common stem cells, which are capable of differentiating toward different cell types of nephron through further involvement of specific transcription factors. Previous studies have suggested the close histogenetic relationship of chromophobe RCC, especially the eosinophilic variant, with oncocytoma. Our finding that the Ksp-cadherin expression was practically the same in these two tumor types supports this relationship.

In summary, Ksp-cadherin is a novel and highly sensitive marker for distal portions of the nephron and their corresponding tumors, that is, chromophobe RCC and oncocytoma. It is eminently applicable in routine pathology practice and can be used in combination with the RCC marker to improve the accuracy of diagnosis of RCC. 


\section{References}

1 Thoenes W, Storkel S, Rumpelt HJ. Human chromophobe cell renal carcinoma. Virchows Arch B Cell Pathol Incl Mol Pathol 1985;48:207-217.

2 Tickoo SK, Amin MB, Zarbo RJ. Colloidal iron staining in renal epithelial neoplasms, including chromophobe renal cell carcinoma: emphasis on technique and patterns of staining. Am J Surg Pathol 1998;22: 419-424.

3 MacLennan GT, Farrow G, Bostwick DG. Immunohistochemistry in the evaluation of renal cell carcinoma: A critical appraisal. J Urol Pathol 1997;6:195-203.

4 Cochand-Priollet B, Molinie V, Bougaran J, et al. Renal chromophobe cell carcinoma and oncocytoma. A comparative morphologic, histochemical, and immunohistochemical study of 124 cases. Arch Pathol Lab Med 1997;121:1081-1086.

5 Avery AK, Beckstead J, Renshaw AA, et al. Use of antibodies to RCC and CD10 in the differential diagnosis of renal neoplasms. Am J Surg Pathol 2000;24:203-210.

6 Martignoni G, Pea M, Chilosi M, et al. Parvalbumin is constantly expressed in chromophobe renal carcinoma. Mod Pathol 2001;14:760-767.

7 Young AN, de Oliveira Salles PG, Lim SD, et al. Beta defensin-1, parvalbumin, and vimentin: a panel of diagnostic immunohistochemical markers for renal tumors derived from gene expression profiling studies using cDNA microarrays. Am J Surg Pathol 2003; 27:199-205.

8 Langner C, Ratschek M, Rehak P, et al. Expression of MUC1 (EMA) and E-cadherin in renal cell carcinoma: a systematic immunohistochemical analysis of 188 cases. Mod Pathol 2004;17:180-188.

9 McGregor DK, Khurana KK, Cao C, et al. Diagnosing primary and metastatic renal cell carcinoma: the use of the monoclonal antibody 'Renal Cell Carcinoma Marker'. Am J Surg Pathol 2001;25:1485-1492.

10 Thomson $\mathrm{RB}$, Igarashi $\mathrm{P}$, Biemesderfer $\mathrm{D}$, et al. Isolation and cDNA cloning of Ksp-cadherin, a novel kidney-specific member of the cadherin multigene family. J Biol Chem 1995;270:17594-17601.

11 Thomson RB, Aronson PS. Immunolocalization of Ksp-cadherin in the adult and developing rabbit kidney. Am J Physiol 1999;277:F146-F156.

12 Thomson RB, Ward DC, Quaggin SE, et al. cDNA cloning and chromosomal localization of the human and mouse isoforms of Ksp-cadherin. Genomics 1998;51:445-451.

13 Kovacs G, Akhtar M, Beckwith BJ, et al. The Heidelberg classification of renal cell tumours. J Pathol 1997; 183:131-133.

14 Storkel S, Eble JN, Adlakha K, et al. Classification of renal cell carcinoma: Workgroup No. 1. Union Internationale Contre le Cancer (UICC) and the American Joint Committee on Cancer (AJCC). Cancer 1997;80: 987-989.

15 Christofori G, Semb H. The role of the cell-adhesion molecule E-cadherin as a tumour-suppressor gene. Trends Biochem Sci 1999;24:73-76.

16 Sobrinho-Simoes M, Oliveira C. Different types of epithelial cadherin alterations play different roles in human carcinogenesis. Adv Anat Pathol 2002;9: 329-337.

17 Berchtold MW. Structure and expression of genes encoding the three-domain $\mathrm{Ca}^{2+}$-binding proteins parvalbumin and oncomodulin. Biochim Biophys Acta 1989;1009:201-215.

18 Pauls TL, Portis F, Macri E, et al. Parvalbumin is expressed in normal and pathological human parathyroid glands. J Histochem Cytochem 2000;48: 105-111. 